\title{
REVIEW
}

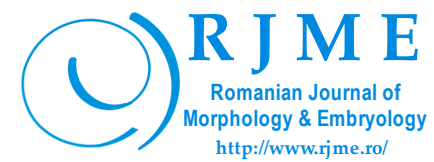

\section{Immunological and hormonal mechanisms in Alzheimer's disease}

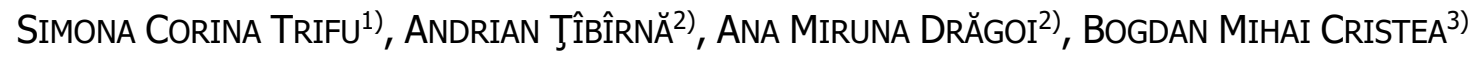 \\ 1) Department of Neurosciences, Carol Davila University of Medicine and Pharmacy, Bucharest, Romania \\ ${ }^{2)}$ Department of Psychiatry, Prof. Dr. Alexandru Obregia Clinical Hospital for Psychiatry, Bucharest, Romania \\ ${ }^{3}$ Department of Morphological Sciences, Carol Davila University of Medicine and Pharmacy, Bucharest, Romania
}

\begin{abstract}
Alzheimer's disease (AD) is a disorder which is today treated and approached at the crossroad of two medical specialties - psychiatry and neurology. The insidious onset which can often mimic depressive disorders or other type of psychiatric disorders, the behavioral changes, the paranoid thoughts usually send people to the psychiatrist, while the brain changes observed on magnetic resonance imaging (MRI) scans and other imaging techniques may indicate the need for neurological monitoring also. The complex symptomatology and progression of this dementia requires a multidisciplinary approach and recent studies focused on adding a third perspective: a metabolic one. The common findings regarding type 2 diabetes and AD made some researchers to informally name it "the third type diabetes". This mini review aims to highlight the mechanisms through which brain insulin resistance can lead to cognitive impairment and to make a short overview of the current findings which demonstrate why insulin may be a promising adjunctive treatment of Alzheimer's dementia, for certain patients.
\end{abstract}

Keywords: schizophrenia, insulin, Alzheimer, diabetes, cognitive impairment.

\section{Introduction}

Alzheimer's disease (AD), as a chronic disorder with a progressive neurodegenerative effect, can be categorized in two types: the one with late-onset and the early-onset one. Patients with early onset are less than $10 \%$ from all the patients suffering of AD. Most of the $50-60 \%$ of population develop AD usually over 60 years, with a severe form of the illness in approximately $10 \%$ over 65 years, all of them with a dramatic life changes in the life of the patient. It is estimated that by 2050 the number of Alzheimer's patients diagnosed globally will be around 140 million. At the same time, a powerful effect lies in the life of the patient's family and friends. This puts an increase pressure on the life of everyone near the patient, from the social, psychical to financial point of view. The increase of the life's expectancy of the population will have a high impact in the number of the patients diagnosed with $\mathrm{AD}$ making the people more aware about this illness and putting a higher social pressure on the society.

\section{a AD and immunological changes}

Efthymiou \& Goate research related to Alzheimer's dementia has focused on identifying microglial clearance of amyloid beta $(\mathrm{A} \beta)$ plaques, that leads to neuronal degeneration through the accumulation in time of $\mathrm{A} \beta$, correlated with the impossibility to regulate the phagocytic function of these cells [1]. At the same time, several recent researches focus on the lifelong neuroinflammation and immunological changes of an Alzheimer's patient; it is known that $\mathrm{AD}$ acts before the detection of clear clinical symptoms. Longitudinal clinical follow-up studies in $\mathrm{A} \beta$ - positive mild cognitive impairment patients have shown a higher risk of converting to AD.

At the same time, Efthymiou \& Goate research highlights a variety of immunity-related genes that make a relative contribution to the development of research related to $\mathrm{AD}$. They are involved in a wide range of immune functions. We refer here to the genes: apolipoprotein $\mathrm{E}$ $(A P O E)$ (the presence of its $\varepsilon 4$ variant - as a risk factor of developing $\mathrm{AD}$ ), bridging integrator 1 (BIN1) (overexpressed in brain for $\mathrm{AD}$ and it also modulate the level of the tau pathway), cluster of differentiation 33 (CD33) (increase expression associated with increased plaque burden), clusterin $(C L U)$, complement receptor 1 (CRl) (its increase is associated with more active microglia), inositol polyphosphate-5-phosphatase D (INPP5D) (expressed in microglia; its high expression is associated with high AD risk), phosphatidylinositol-binding clathrin assembly protein $(P I C A L M)$ (involved in clathrin-mediated endocytosis that is an important step in the intracellular trafficking of proteins linked with neuronal function through synaptic vesicle cycling), phospholipase $\mathrm{C}$ gamma 2 (PLCG2) (expressed in brain microglia; low activation gives protection against $\mathrm{AD}$ ), membrane spanning 4-domains A6A (MS4A6A) (mouse ortholog to MS4A6A - selectively expressed in myeloid cells and implicated in AD) and triggering receptor expressed on myeloid cells 2 (TREM2) (associated with low binding apolipoproteins, including CLU). These genes were identified in whole genome sequences and genome-wide association study (GWAS) analyzes $[1,2]$. Recent Fox et al. study has established a link between the risk of developing $\mathrm{AD}$ and the time a woman spends cumulatively during all the pregnancies

This is an open-access article distributed under the terms of a Creative Commons Attribution-NonCommercial-ShareAlike 4.0 International Public License, which permits unrestricted use, adaptation, distribution and reproduction in any medium, non-commercially, provided the new creations are licensed under identical terms as the original work and the original work is properly cited. 
she has had. Throughout life, the immunological changes that take place, especially in the first trimester of pregnancy, protect the woman so that in the future she reduces her risk of developing Alzheimer's dementia. The pathophysiology of Alzheimer's dementia is associated with immunological changes and systemic inflammatory processes, as well as increased proliferation of regulatory T-cells (Treg). These are usually modulators, which modify the immune system, making it more adaptive, thus protecting the one in question from the early development of Alzheimer's dementia [3]. Treg cells play a key role in maintaining pregnancy, by supporting tissue hemostasis and by increasing immune tolerance. This is not the case when it comes to "toxic pregnancies". Neuroendocrine changes are directly related to the onset of $\mathrm{AD}$ by increased cytotoxic activity and increased tumor necrosis factoralpha (TNF- $\alpha$ ), pathophysiological conditions that lead to decreased cognitive function of the patient (deficient actions in anticipation, planning, sequencing, operationalization and retroactive feedback). Morphopathologically, we are dealing with the reduction of brain volume in the hippocampus and temporal lobes, while from an immunological point of view, there are immuno-neuroendocrine changes [4]. From clinical symptomatology point of view, we are talking about the progressive decrease of the capacity to operationalize on abstract terms and on generalization terms, with reduction to the functioning in concrete and particular life area.

The adaptive immune system is ruled by the $\mathrm{T}$ - (that gives a cell-mediated response) and B- (that initiate an antibody response) lymphocyte cells. The activation of the adaptive immune system seen through significant modifications in frequencies of lymphocyte subpopulations, complicated by crossing the blood-brain barrier (BBB) through the release of cytokines by the effector T-helper (Th) cell immune response, trigger the events leading to neuroinflammation. In this way, increased frequencies of Th1 and Th17 cells indicate a pro-inflammatory signature for neurodegenerative $\mathrm{AD}$ [5]. Th1 releases interferongamma (IFN- $\gamma$ ) that has a neurotoxic effect mediated by activated microglia in AD patients and Th17 releases interleukin-17 (IL-17) cytokine - also directly associated with the risk of cognitive impairment. The limitations of such studies come from the fact that they are performed on postmortem subjects or on animals, mainly mice.

Older adults go through a large number of immunological changes: thymus involution, decreased production of T-lymphocytes, impairment of pre-existing lymphocytes, impaired functioning of B-lymphocytes, cytokines, decreased killer macrophage cells. All these changes demonstrate a decrease in the ability of the immune system to counteract diseases, be they infectious, multipliers, karyogenesis and neoplasms, neurodegeneration. Changes in neuroendocrine functions cause changes in bidirectional communication between the immune system and the endocrine system. Such concepts, such as immune senescence or endocrine senescence, are current. Both can be caused by psychosocial stressors, trauma, abuse, mourning or seem to be the consequence of declining adaptive internal resources. At the same time, decreases the capacity of defense mechanisms to cope, reduces the network of social or family support, decreases community support for the elderly. The feelings of anxiety return with the fear of the irreversibility of the loss of cognitive functions, but also of emotional losses of loved ones in everyday life. This is frustrating and frightening for patients who are still aware of their memory loss. In the American literature, this brings into question differential diagnoses, such as dissociative identity disorder [6]. We are talking about transient amnesias, the mechanism being also of dissociative order in Alzheimer's dementia, but the neurodegenerative nuance predominates, while in dissociative pathologies, the importance of stressors predominates when they are starting at younger ages.

The oxidative stress that precedes the AD damage, present also at the normal people during ageing, is due to oxidizing free radicals released by a dysfunctional mitochondrion. $\mathrm{A} \beta$ is the prime initiator of oxidative damage. The $\mathrm{A} \beta$ is generated after enzymatic cleavage by $\beta$ - and $\gamma$-secretases of the amyloid precursor protein that is expressed especially in the central nervous system (CNS). It has a leading role in the development of the brain, memory, and synaptic plasticity. The increase levels of $\mathrm{A} \beta$ associated with an increase level of oxygenation levels from protein, lipids and nucleic acids have been identified in hippocampus and cortex. In hippocampus, the increased level of $\mathrm{A} \beta$ has been identified through the oxidizing of low-density lipoprotein receptor-related protein 1 (LRPl) protein that is involved in the efflux that crosses the BBB from the brain to the blood $[7,8]$.

Age-related changes in the immune system are common and known as immune senescence. With increasing age, the body becomes more sensitive to infections, inflammation, wound healing. Thus, Alzheimer's dementia reaches the top five as the cause of death, after heart disease, respiratory disease, stroke. This suggests the difficulties faced by relatives in caring for the elderly, especially those with $\mathrm{AD}$ or other forms of dementia. This becomes important due to the increased emotional stress of both the patient and the caregiver, not considering the physical stress of the person with $\mathrm{AD}$. The caregiver or support person may experience a burnout syndrome, even if they start from the idea that they are caring for a formal and emotionally uninvolved patient. However, the correlations with the depressive or anxious symptoms of the elderly patient, his neuroendocrine changes, affect the quality of life of both the patient and his relatives [9]. Not infrequently there are abusive behaviors at the limit, which are explained by the perspective of neurohormonal or chemical neuromediators involved. In AD, the boundary between the neurohormonal and neurobiochemical component is fragile, compared to translated role models, attitudes and behaviors, emotions, primitive anxieties that awaken in the patient and that lead to abusive attitudes on the part of caregivers [10].

\section{ㅁ Linking AD to diabetes}

The link between AD and type 2 diabetes has been extensively researched. Age-related AD is the leading cause of dementia. As the years go by, we speak of a neurodegenerative disorder with an insidious onset, which first affects the fixation memory, then followed by performance decreases in the area of attention and 
language, until the patient completely loses its functionality. By the time, the symptoms become apparent to those around them, the changes in the brain are already irreversible, and the available medications are only aimed at delaying subsequent losses. In other words, there is no anti-dementia medication to restore neural circuits and their functionality. In terms of antidementia medication, it increases the functioning of neurons that remain intact, by establishing new synapses and new functional circuits, available to the use of resources in terms of memory. The more complex the intellectual capacity of the patient with Alzheimer's dementia and the higher his social functioning, the more the person in question will make up for the precarious state of fixation by complex gnostic functions. Paraclinical investigations reveal neuropathological markers related to $\mathrm{AD}: \beta$ extracellular amyloid plaques, neurofibrillary disorder, impaired synaptic transmission, inflammation, neuronal destruction [11].

Increased insulin blood levels present in patients with type 2 diabetes promote $\mathrm{A} \beta$ accumulation (that leads to neuronal degeneration, as mentioned before). Mitochondrial dysfunction, that has a key role in the pathogenesis of type 2 diabetes and $\mathrm{AD}$ at the same time, precedes and sustains $\mathrm{A} \beta$ accumulation. $\mathrm{A} \beta$ interacts with mitochondria proteins, disrupting the electron transport chain and promoting mitochondrial dysfunction and an increased generation of reactive oxygen species (with a major role in signaling pathways) [12]. Type 2 diabetes causes chronic inflammation, oxidative stress, microvascular disease, hyperinsulinemia, all of which ultimately lead to advanced impairment of glycosylation processes, which play a significant role in the pathology of AD [13].

A recent meta-analysis of patients with type 2 diabetes highlights a high risk for both Alzheimer's dementia and vascular dementia - 56\% increased risk for developing $\mathrm{AD}$ and $127 \%$ increased risk for developing a vascular dementia [14]. At the same time, another meta-analysis of clinical trials showed that type 2 diabetes increases the risk for $\mathrm{AD}$, without any correlation with the pathology of obesity [15].

Positron emission tomography-computed tomography (PET-CT) scans with typical translocator protein radiotracers shows the relationship between $\mathrm{A} \beta$ accumulation and microglia activation during disease evolution in time. Also, it can be made a measurement of inflammation which shows high binding in parietal and temporal cortices in AD patients. Neuroimaging over extender period of time have associated $\mathrm{AD}$ with pathophysiological changes prior to cognitive symptoms [16].

New directions of research involve patients who may have human-induced pluripotent stem cells (hiPSC) transplanted. These situations, despite the limitations caused by the significant decrease in the number of neurons already affected, bring clinically significant results in dopaminergic neurotransmitters. Thus, AD involves genetically determined mechanisms to ontogenetically conditioned mechanisms and, further, molecular mechanisms. We noted that PSC transplantation amplifies dopaminergic transmission and diminishes the effects of neuronal destruction in AD [17].
Insulin resistance of the brain appears to be caused by a decrease in insulin receptor expression, as well as a decrease in kinase receptor activity, all of which are related to hyperinsulinemia associated with type 2 diabetes [18]. Some pathways that are involved in the pathogenesis of $\mathrm{AD}$ may be altered due to insulin degradation. We mention here protein kinase $\mathrm{B}(\mathrm{PKB})$, mitogen-activated protein kinase (MAPK) and glycogen synthetase kinase 3 beta $(\mathrm{GSK} 3 \beta)$. The expression of MAPK is related to the formation of $\mathrm{A} \beta$ and neurofibrillary disorder. MAPK is elevated in patients with Alzheimer's dementia. P38 MAPKs are a class of MAPKs that are receptive to stress stimuli, such as cytokines, ultraviolet irradiation, thermal and osmotic shock. Persistent activation of p38 MAPK in muscle satellite cells (stem cells) because of aging affects muscle regeneration. P38 MAPKs participate in signaling the control of cellular responses to cytokines and stress. They are involved in cell differentiation, apoptosis and autophagy. If insulin resistance is present, GSK $3 \beta$ can be inhibited by signaling PKB. This leads to dephosphorylation and activation of glycogen synthase in glycogenesis (Cori cycle) eventually leading to hyperphosphorylation of tau protein. Tau protein, under normal conditions, stabilizes neuronal microtubules. Under pathological conditions, tau protein can generate neuronal toxicity. We can thus conclude that cognitive impairment can be addressed by exogenous insulin intake [19].

Postmortem studies performed on the brains of patients diagnosed with Alzheimer's dementia, support the insufficient signaling of the moment of insulin transmission in the synaptic cleft.

\section{a Antidiabetic medication used in AD}

Despite the social impact and huge costs of supporting $\mathrm{AD}$ societies, the number of patients with $\mathrm{AD}$ continues to rise. For more than a decade, not many new therapies have been approved. Clinical trials have been performed with single-agent therapies, which do not always stop the disease or prevent the development of new symptoms compared to placebo.

Type 2 diabetes is a risk factor for Alzheimer's dementia. Glucagon-like peptide-1 (GLP-1) is a 30-unit endogenous peptide hormone that is involved in the regulation of insulin. GLP-1 receptor agonists act as a growth factor for dendrites, but also as a factor that reduces oxidative stress on neurons. The receptors themselves play a significant role in memorization, storage, evocation and learning in general. A variety of therapies have been developed that extend the half-life (normally rapidly) of local GLP-1, which would include: Exenatide, Liraglutide, Lixisenatide and Albiglutide (a GLP-1 dimer fused to human albumin).

Liraglutide is a blood-permeable barrier peptide, an analogue of GLP-1 used to treat type 2 diabetes. Liraglutide was able to restore learning in a significantly superior way compared to the control group. Liraglutide corrected longterm hippocampal areas in the cornu Ammonis 1 (CA1) region in $\mathrm{A} \beta \mathrm{PP} / \mathrm{PS} 1$ transgenic mice. After eight weeks, $\mathrm{A} \beta$ plaques in the cortex were reduced by $50 \%$, while Congo Red stained plaques were reduced by $25 \%$ [20]. 
Tryptophan metabolism is largely due to the degradation of kynurenine. Kynurenine 3-monooxygenase (KMO) produces metabolites that induce excitotoxicity by $N$ methyl-D-aspartate (NMDA) receptor agonism and, as such, high ratios of kynurenine (a metabolite of Ltryptophan amino acid used in the production of niacin) and tryptophan are associated with neurodegeneration. A KMO inhibitor, as well as JM6, has been shown to increase synaptophysin levels in an $\mathrm{A} \beta \mathrm{PP}$-transgenic mice model by preventing synapse loss in AD. In contrast, they had no effect on amyloid plaque loading or on significant improvement in cognitive function. For example, spatial memory has been improved, but not spatial learning [21] The clinical correspondent of these neurophysiological and morphopathological disorders involves the use of selective serotonin reuptake inhibitors (SSRIs) or Tianeptine or Trazodone antidepressants in AD [22].

Empagliflozin (EMP) is a co-transporter of sodiumglucose inhibitor used as an antidiabetic agent. It controls hyperglycemia and reduces cardiovascular comorbidities or deaths associated with metabolic disorders in type 2 diabetes. EMP appears to decrease cortical reduction as well as neuronal loss in treated mice. Clinical trials in EMP-treated mice also showed a decrease in bleeding and improved microglial function, amyloid-laden senile plaques were lower, and effects were found to improve cognitive deficits in APP/PS1xdb/db mice [23].

As shown in the studies mentioned above, $\mathrm{AD}$ is linked to insulin resistance of the CNS. Abnormalities in insulin receptor expression led to low insulin levels. The cognitive impairment associated with this dementia is thus caused by deficits in insulin signaling at the synaptic level. This finding leads more and more researchers to conduct studies on the role of insulin in cognitive performance in healthy people compared to cognitive performance among patients with AD.

Cerebrospinal fluid (CSF) has long been known to have appreciable concentrations of glucose and insulin, reflecting only partially the serum glucose and insulin levels. In humans, the transfer of insulin from the blood to the CSF has been monitored during intravenous insulin injections. Interestingly, obesity decreases the CSF/plasma ratio in terms of insulin levels. Similarly, CSF/plasma ratios for leptin and adiponectin are also low. Homeostasis model assessment of insulin resistance (HOMA-IR) (a method for the determination of serum insulin) was used which showed correlations between elevated CSF levels of Alzheimer's dementia biomarkers: soluble amyloid precursor protein beta $(\operatorname{sAPP} \beta)$, tau phosphorylated at threonine 181 (P-tau 181), with $A P O E \varepsilon 4$ carriers [24]. In addition, elevated insulin levels in the CSF correlate with poor cognitive performance in patients with type 2 diabetes and AD.

The only therapeutic effect of peripherally administered insulin is to lower blood glucose. Peripherally administered insulin does not cross the BBB and does not reach the CNS. Therefore, another way of administering insulin had to be found to reach the brain directly, short-circuiting the BBB.

Consequently, the intranasal method of administering insulin makes it reach directly from the nostrils into the brain. Such clinical studies have been performed on both healthy people in terms of cognitive function and people with AD. A significant increase in memory and learning processes has been reported in subjects receiving intranasal insulin [25].

Another study in healthy adults showed that individuals who were exposed to higher insulin infusion rates had increased memory improvements, greater cognitive flexibility, and increased attention span, such as concentration, stability, and more selectivity and distributivity (via the Stroop test) [26].

One of the best-known studies in this field is the one led by Dr. Suzanne Craft. One hundred forty adults were included in a randomized, double-blind, placebocontrolled study. Selected patients had either mild cognitive impairment or symptoms of mild or moderate Alzheimer's dementia [27]. The protocol lasted four months, during which time some of the subjects received placebo medication, others of them a low dose of intranasal insulin 20 units and the third batch, a high dose of intranasal insulin 40 units. Memory and cognitive performance were assessed to determine the efficacy of intranasal insulin and to determine whether the effects were dose dependent. Patients who received a lower dose had a significant improvement in memory compared to the placebo group. There was also good cognitive functioning for both groups of patients who received insulin compared to those who received placebo.

Using neuroimaging techniques, it was observed at the end of the study that in the group of subjects who received placebo there was a lower use of glucose in the cerebral cortex. This indicated that the neurodegeneration is progressive. The group of patients treated with intranasally administered insulin had a preservation of brain metabolism, showing that this therapy can stop or at least slow down the losses caused by Alzheimer's dementia.

However, in the same paper, it is presented that individuals who had the $A P O E \& 4$ allele (a genetic predictor of the disease) had a significantly smaller improvement compared to those who did not have this allele [24].

\section{ㅁ Conclusions}

$\mathrm{AD}$ is a debilitating burden, but also socially, medically and economically. Any therapeutic option that can prevent it, delay progress or reverse the terrible effects of this disease would be very successful. The complex physiology of Alzheimer's dementia may require combined treatments rather than monotherapy. However, reliable evidence refers to the brain's resistance to insulin, which plays a major role in the pathogenesis of AD. This is generally compatible with the "amyloid approach". The correlation between $\mathrm{AD}$ and type 2 diabetes has been studied for years. Insulin does much more than regulate blood sugar, as is commonly known. Insulin is a crucial "growth factor" for neurons. Lack of insulin makes neurons vulnerable, which eventually leads to neuronal apoptosis. Even though $\mathrm{AD}$ is mainly related to age, current studies and modern trends in medicine tell us that cognitive impairment is not a normal part of aging. This is supported by the promising 
results of studies on ancillary treatments for $\mathrm{AD}$, but no treatment is "suitable for everyone". At present, several clinical studies on the authorization of nasal insulin sprays are active. Intranasal administration of insulin, which allows it to reach the brain directly, has also been chosen as an important therapeutic strategy by the National Institutes of Health (NIH) in the United States. It receives significant funding for research, with the hope that a good enough way to prevent or treat AD by 2025 will be studied and developed.

\section{Conflict of interests}

The authors declare that they have no conflict of interests.

\section{References}

[1] Efthymiou AG, Goate AM. Late onset Alzheimer's disease genetics implicates microglial pathways in disease risk. Mol Neurodegener, 2017, 12(1):43. https://doi.org/10.1186/s130 24-017-0184-x PMID: 28549481 PMCID: PMC5446752

[2] Harold D, Abraham R, Hollingworth P, Sims R, Gerrish A, Hamshere ML, Pahwa JS, Moskvina V, Dowzell K, Williams A, Jones N, Thomas C, Stretton A, Morgan AR, Lovestone S, Powell J, Proitsi P, Lupton MK, Brayne C, Rubinsztein DC, Gill M, Lawlor B, Lynch A, Morgan K, Brown KS, Passmore PA, Craig D, McGuinness B, Todd S, Holmes C, Mann D, Smith AD, Love S, Kehoe PG, Hardy J, Mead S, Fox N, Rossor M, Collinge J, Maier W, Jessen F, Schürmann B, Heun R, van den Bussche H, Heuser I, Kornhuber J, Wiltfang J, Dichgans M, Frölich L, Hampel H, Hüll M, Rujescu D, Goate AM, Kauwe JS, Cruchaga C, Nowotny P, Morris JC, Mayo K, Sleegers K, Bettens K, Engelborghs S, De Deyn PP, Van Broeckhoven C, Livingston G, Bass NJ, Gurling $H$, McQuillin A, Gwilliam R, Deloukas $P$, Al-Chalabi A, Shaw CE, Tsolaki M, Singleton AB, Guerreiro R, Mühleisen TW, Nöthen MM, Moebus S, Jöckel KH, Klopp N, Wichmann HE, Carrasquillo MM, Pankratz VS, Younkin SG, Holmans PA, O'Donovan M, Owen MJ, Williams J. Genome-wide association study identifies variants at $C L U$ and PICALM associated with Alzheimer's disease. Nat Genet, 2009 41(10):1088-1093. https://doi.org/10.1038/ng.440 PMID: 19734902 PMCID: PMC2845877

[3] Fox M, Berzuini C, Knapp LA, Glynn LM. Women's pregnancy life history and Alzheimer's risk: can immunoregulation explain the link? Am J Alzheimers Dis Other Demen, 2018, 33(8): 516-526. https://doi.org/10.1177/1533317518786447 PMID: 30060670 PMCID: PMC6442681

[4] Tublin JM, Adelstein JM, Del Monte F, Combs CK, Wold LE. Getting to the heart of Alzheimer disease. Circ Res, 2019 124(1):142-149. https://doi.org/10.1161/CIRCRESAHA.118. 313563 PMID: 30605407 PMCID: PMC6319653

[5] Sommer A, Winner B, Prots I. The Trojan horse - neuroinflammatory impact of $T$ cells in neurodegenerative diseases. Mol Neurodegener, 2017, 12(1):78. https://doi.org/10.1186/ s13024-017-0222-8 PMID: 29078813 PMCID: PMC5658940

[6] Trifu S. Dissociative identity disorder. Psychotic functioning and impairment of growing-up processes. J Educ Sci Psychol, 2019, 9/71(2):102-108. http://jesp.upg-ploiesti.ro/index.php? option=com phocadownload\&view=category\&id=32:journal -vol-ix-|xxi-no-22019\&ltemid=16

[7] Nguyen HN, Byers B, Cord B, Shcheglovitov A, Byrne J, Gujar P, Kee K, Schüle B, Dolmetsch RE, Langston W, Palmer TD, Pera RR. LRRK2 mutant iPSC-derived DA neurons demonstrate increased susceptibility to oxidative stress. Cell Stem Cell, 2011, 8(3):267-280. https://doi.org/10.1016/j.stem. 2011.01.013 PMID: 21362567 PMCID: PMC3578553

[8] Cheignon C, Tomas M, Bonnefont-Rousselot D, Faller P, Hureau C, Collin F. Oxidative stress and the amyloid beta peptide in Alzheimer's disease. Redox Biol, 2018, 14:450-464. https://doi.org/10.1016/j.redox.2017.10.014 PMID: 29080524 PMCID: PMC5680523

[9] Yu H, Wang X, He R, Liang R, Zhou L. Measuring the caregiver burden of caring for community-residing people with Alzheimer's disease. PLoS One, 2015, 10(7):e0132168. https:// doi.org/10.1371/journal.pone.0132168 PMID: 26154626 PMCID: PMC4496054

[10] Alves LCS, Monteiro DQ, Bento SR, Hayashi VD, Pelegrini LNC, Vale FAC. Burnout syndrome in informal caregivers of older adults with dementia: a systematic review. Dement Neuropsychol, 2019, 13(4):415-421. https://doi.org/10.1590/1980 -57642018dn13-040008 PMID: 31844495 PMCID: PMC6907708

[11] Querfurth HW, LaFerla FM. Alzheimer's disease. N Engl J Med, 2010, 362(4):329-344. https://doi.org/10.1056/NEJMra0909 142. Erratum in: N Engl J Med, 2011, 364(6):588. PMID: 20107219

[12] Jack CR Jr, Knopman DS, Jagust WJ, Shaw LM, Aisen PS, Weiner MW, Petersen RC, Trojanowski JQ. Hypothetical model of dynamic biomarkers of the Alzheimer's pathological cascade. Lancet Neurol, 2010, 9(1):119-128. https://doi.org/10.1016/S 1474-4422(09)70299-6 PMID: 20083042 PMCID: PMC2819840

[13] de la Monte SM, Wands JR. Alzheimer's disease is type 3 diabetes-evidence reviewed. J Diabetes Sci Technol, 2008, 2(6):1101-1113. https://doi.org/10.1177/1932296808002006 19 PMID: 19885299 PMCID: PMC2769828

[14] Bellou V, Belbasis L, Tzoulaki I, Middleton LT, loannidis JPA, Evangelou $E$. Systematic evaluation of the associations between environmental risk factors and dementia: an umbrella review of systematic reviews and meta-analyses. Alzheimers Dement, 2017, 13(4):406-418. https://doi.org/10.1016/j.jalz.2016.07. 152 PMID: 27599208

[15] Profenno LA, Porsteinsson AP, Faraone SV. Meta-analysis of Alzheimer's disease risk with obesity, diabetes, and related disorders. Biol Psychiatry, 2010, 67(6):505-512. https://doi. org/10.1016/j.biopsych.2009.02.013 PMID: 19358976

[16] Nordberg A. Dementia in 2014. Towards early diagnosis in Alzheimer disease. Nat Rev Neurol, 2015, 11(2):69-70. https:// doi.org/10.1038/nrneurol.2014.257 PMID: 25623789

[17] de Leeuw S, Tackenberg C. Alzheimer's in a dish - induced pluripotent stem cell-based disease modeling. Transl Neurodegener, 2019, 8:21. https://doi.org/10.1186/s40035-019-01 61-0 PMID: 31338163 PMCID: PMC6624934

[18] Kim B, Sullivan KA, Backus C, Feldman EL. Cortical neurons develop insulin resistance and blunted Akt signaling: a potential mechanism contributing to enhanced ischemic injury in diabetes. Antioxid Redox Signal, 2011, 14(10):1829-1839. https://doi.org/ 10.1089/ars.2010.3816 PMID: 21194385 PMCID: PMC3078499

[19] Edwards III GA, Gamez N, Escobedo G Jr, Calderon O, Moreno-Gonzalez I. Modifiable risk factors for Alzheimer's disease. Front Aging Neurosci, 2019, 11:146. https://doi.org/ 10.3389/fnagi.2019.00146 PMID: 31293412 PMCID: PMC 6601685

[20] Hansen $\mathrm{HH}$, Fabricius $\mathrm{K}$, Barkholt $\mathrm{P}$, Kongsbak-Wismann $\mathrm{P}$, Schlumberger C, Jelsing J, Terwel D, Termont A, Pyke C, Knudsen LB, Vrang N. Long-term treatment with liraglutide, a glucagon-like peptide-1 (GLP-1) receptor agonist, has no effect on $\beta$-amyloid plaque load in two transgenic APP/PS1 mouse models of Alzheimer's disease. PLoS One, 2016, 11(7):e0158205. https://doi.org/10.1371/journal.pone.0158205 PMID: 27421117 PMCID: PMC4946784

[21] Campbell BM, Charych E, Lee AW, Möller T. Kynurenines in CNS disease: regulation by inflammatory cytokines. Front Neurosci, 2014, 8:12. https://doi.org/10.3389/fnins.2014.00 012 PMID: 24567701 PMCID: PMC3915289

[22] Ashford JW. Treatment of Alzheimer's disease: trazodone, sleep, serotonin, norepinephrine, and future directions. J Alzheimers Dis, 2019, 67(3):923-930. https://doi.org/10.3233/ JAD-181106 PMID: 30776014 PMCID: PMC6398534

[23] Hierro-Bujalance C, Infante-Garcia C, Del Marco A, Herrera M, Carranza-Naval MJ, Suarez J, Alves-Martinez P, Lubian-Lopez S, Garcia-Alloza M. Empagliflozin reduces vascular damage and cognitive impairment in a mixed murine model of Alzheimer's disease and type 2 diabetes. Alzheimers Res Ther, 2020, 12(1):40. https://doi.org/10.1186/s13195-020-00607-4 PMID: 32264944 PMCID: PMC7140573

[24] Niemantsverdriet E, Valckx S, Bjerke M, Engelborghs S. Alzheimer's disease CSF biomarkers: clinical indications and rational use. Acta Neurol Belg, 2017, 117(3):591-602. https://doi.org/10.1007/s13760-017-0816-5 PMID: 28752420 PMCID: PMC5565643

[25] Reger MA, Watson GS, Frey WH 2nd, Baker LD, Cholerton B, Keeling ML, Belongia DA, Fishel MA, Plymate SR, Schellenberg GD, Cherrier MM, Craft S. Effects of intra- 
nasal insulin on cognition in memory-impaired older adults: modulation by APOE genotype. Neurobiol Aging, 2006, 27(3): 451-458. https://doi.org/10.1016/j.neurobiolaging.2005.03.016 PMID: 15964100

[26] Kern W, Peters A, Fruehwald-Schultes B, Deininger E, Born J, Fehm HL. Improving influence of insulin on cognitive functions in humans. Neuroendocrinology, 2001, 74(4):270-280. https:// doi.org/10.1159/000054694 PMID: 11598383
[27] Craft S, Baker LD, Montine TJ, Minoshima S, Watson GS, Claxton A, Arbuckle M, Callaghan M, Tsai E, Plymate SR, Green PS, Leverenz J, Cross D, Gerton B. Intranasal insulin therapy for Alzheimer disease and amnestic mild cognitive impairment: a pilot clinical trial. Arch Neurol, 2012, 69(1): 29-38. https://doi.org/10.1001/archneurol.2011.233 PMID: 21911655 PMCID: PMC3260944

\section{Corresponding authors}

Ana Miruna Drăgoi, Department of Psychiatry, Prof. Dr. Alexandru Obregia Clinical Hospital for Psychiatry, 10 Berceni Highroad, Sector 4, 041914 Bucharest, Romania; Phone +40770-420 108, e-mail: anamirunadragoi@gmail.com

Simona Corina Trifu, Lecturer, PhD, Department of Neurosciences, Carol Davila University of Medicine and Pharmacy, 37 Dionisie Lupu Street, Sector 2, 020021 Bucharest, Romania; Phone +40731-581 100, Fax +4031-105 58 90, e-mail: simona.trifu@umfcd.ro

Received: November 12, 2020

Accepted: May 16, 2021 